\title{
LncRNA DNAJC3-ASI Promotes Hepatocellular Carcinoma (HCC) Progression via Sponging Premature miR-27b
}

\author{
Changbo $\mathrm{Fu}^{\prime}$ \\ Jianxiu $\mathrm{Li}^{2}$ \\ Ping $\mathrm{Li}^{3}$ \\ Dan Cheng ${ }^{4}$
}

\begin{abstract}
'Department of Hepatobiliary and Pancreatic Surgery, Hubei Cancer Hospital, Wuhan City, 430000, People's Republic of China; ${ }^{2}$ Disinfection Supply Center of Weifang Yidu Central Hospital, Weifang City, 266000, People's Republic of China; ${ }^{3}$ Emergency Care Center of Qingdao Central Hospital, Qingdao City, 266000, People's Republic of China;

${ }^{4}$ Department of Liver Disease Infection, Edong Healthcare City Hospital of

Traditional Chinese Medicine, Infections Disease Hospital, Huangshi City, 435000, People's Republic of China
\end{abstract}

Purpose: Long non-coding RNA (lncRNA) DNAJC3 antisense RNA 1 (head to head) (DNAJC3-AS1) plays a key role in the progression of several cancers. However, its biological role in hepatocellular carcinoma (HCC) is still unclear. We aimed to investigate the role of DNAJC3-AS1 in the development of HCC and reveal the potential mechanisms.

Materials and Methods: Expression analysis of DNAJC3-AS1 and microRNA-27b (miR$27 \mathrm{~b}$ ) at both mature and premature levels was determined by RT-qPCR. HCC patients were followed up for 5 years to analyze the prognostic value of DNAJC3-AS1 for HCC. The direct interaction between DNAJC3-AS1 and premature miR-27b was analyzed with RNA pull-down assay. Subcellular analysis of DNAJC3-AS1 was explored by subcellular fractionation assay. DNAJC3-AS1 overexpression and knockdown were carried out to analyze the role of DNAJC3-AS1 in miR-27b maturation. Cell proliferation was analyzed by BrdU assay.

Results: DNAJC3-AS1 was overexpressed in HCC and predicts the poor survival. MiR-27b was downregulated at mature miRNA level, but upregulated at premature level. DNAJC3AS1 directly interacted with premature miR-27b and was localized to both nuclear and cytoplasm. DNAJC3-AS1 overexpression upregulated premature miR-27b and downregulated mature miR-27b, while DNAJC3-AS1 knockdown led to the opposite results. DNAJC3-AS1 suppressed the role of miR-27b in inhibiting cell proliferation.

Conclusion: DNAJC3-AS1 promotes HCC by sponging premature miR-27b and might be a biomarker and therapeutic target for HCC.

Keywords: DNAJC3-AS1, miR-27b, hepatocellular carcinoma, maturation

\section{Introduction}

Hepatocellular carcinoma (HCC), also referred to as hepatoma, is a type of malignant neoplasm that originates from hepatocytes in the liver. ${ }^{1,2}$ Although HCC is the fifth most common cancer in males and seventh in females, it is the second leading causes of cancer deaths in both genders, mainly owing to its extremely aggressive nature and poor prognosis. ${ }^{3}$ According to the latest global statistics, the median survival time of HCC is only about 6-20 months, and only less than $10 \%$ of HCC patients can survive 5 years after initial diagnosis. ${ }^{4,5}$ More than $90 \%$ of HCC cases occur in cirrhosis patients, and the major cause is alcohol. ${ }^{6}$ However, molecular mechanism of HCC remains unclear, which limits the development of novel diagnostic and treatment approaches for HCC. ${ }^{7}$
Correspondence: Dan Cheng

Department of Liver Disease Infection, Edong Healthcare City Hospital of

Traditional Chinese Medicine, Infections

Disease Hospital, No. 634 Shenxia Road, Huangshi City, 435000, People's Republic of China

Email chengdandisease@I63.com 
Although current treatment approaches for HCC, such as surgeries (liver transplantation, cryoablation and resections) and nonsurgical strategies (such as external beam radiation therapy, percutaneous ethanol injection, and chemotherapy), have significantly improved the survival of HCC patients in the past centuries, especially the survival condition of advanced HCC patients, ${ }^{8}$ no significant advances have been achieved in recent years. $^{9,10}$ Focusing on regulating gene expression involved in cancer biology, targeted therapy is an emerging novel approach for cancer treatment. ${ }^{11,12}$ However, more effective targets with high safety remain needed. The major role of long non-coding RNAs (lncRNAs) is to regulate gene expression rather than coding proteins, suggesting that lncRNAs are promising targets for anticancer therapy. ${ }^{13,14}$ DNAJC3 antisense RNA 1 (head to head) (DNAJC3-AS1) accelerates the progression of several cancers, ${ }^{15-17}$ but the participation of this lncRNA in $\mathrm{HCC}$ is unclear. We predicted that DNAJC3-AS1 may interact with miR-27b, which is a cancer-related miRNA in cancers. ${ }^{18}$ The function of miR-27b in cancers and the molecular mechanisms that mediate its function have been widely studies, ${ }^{18}$ while the upstream regulators of miR-25b in cancer biology are unclear. We therefore speculated that DNAJC3-AS1 could interact with miR$27 \mathrm{~b}$ to participate in cancer biology. This study aimed to explore the involvement of DNAJC3-AS1 in HCC, with a focus on its interaction with miR-27b.

\section{Materials and Methods}

\section{Patients and Cell Lines}

Paired HCC and non-tumor tissues were donated by a total of $66 \mathrm{HCC}$ patients at Edong Healthcare City Hospital of Traditional Chinese Medicine, Infection Disease Hospital (Ethics Committee approved this study). All patients underwent surgical resection of the primary tumors. Tumor tissues were dissected by histopathologists to separate paired tissue samples, which were immediately transferred to liquid nitrogen for storage. Patients' clinical data are presented in Table 1. All patients signed informed consent.

Two human HCC cell lines HepG2 and Huh7 (ATCC) were used to perform cell transfections and other cell experiments. These HCC cell lines were cultured in DMEM medium supplemented with $10 \%$ fetal bovine serum (FBS), 100 units $/ \mathrm{mL}$ penicillin and $100 \mu \mathrm{g} / \mathrm{mL}$ streptomycin. All cells were cultured in an atmosphere at $37^{\circ} \mathrm{C}$ with $5 \% \mathrm{CO}_{2}$.

\section{Follow-Up Analysis}

Since the day of discharge, all 66 patients were visited in a monthly manner for a total of 5 years to study the prognostic value of DNAJC3-AS1 for HCC. Follow-up was carried out through telephone or outpatient visit in rare cases. No patient died of cases other than HCC during follow-up. No patient was lost during the follow-up periods.

\section{Cell Transfections}

HepG2 and Huh7 cells were transfected with DNAJC3AS1, si-DNAJC3-AS1, or miR-27b mimic using Neon Electroporation Transfection device (Thermo Fisher Scientific) according to the manufacturer's protocol. Cells cultured in fresh medium without transfections until the end of experiment were served as the control (C). The sequence of MiR-27b mimics was (5'TTCACAGTGGCTAAGTTCTGCAA-3'), and the target sequences of DNAJC3-AS1 siRNA were ggugcugaauguggaguaatt (F) and uuacuccacauucagcacett (R).

NucleoSpin RNA Plus XS (Takara Bio) was used to isolate total RNA from paired tissues and cells of HepG2 and Huh7 cell lines. To reduce DNA contamination, all RNA samples were incubated with DNase I (NEB) to achieve a ratio of OD260/280 above 1.8. Bioanalyzer was used to make sure all RNA samples were with a RIN value higher than 8.0.

\section{RT-qPCRs}

Total RNA (3500 ng) was used as template to prepare cDNA samples through reverse transcriptions. With cDNA samples as template, qPCRs were performed to determine the expression of DNAJC3-AS1 and miR-27b with $18 \mathrm{~S}$ rRNA and U6 as endogenous control, respectively. Comparative $\mathrm{Ct}$ method was used to analyze $\mathrm{Ct}$ values and normalize gene expression levels. The primer sequences were DNAJC3-AS1 forward 5'-AGCGA TTGTGGAAGA CCCT-3' and reverse 5'-TTTCCCCTGG TAAGCGCAA-3', premature miR-27b forward $5^{\prime}$-ACCTC TCUAACAAGGTGCAG- $3^{\prime}$ and $5^{\prime}$-CACCTTCTCTTC AGGTGC-3', U6 forward 5'-CTCGCTTCG GCAGCACA-3' and reverse 5'-AACGCTTCACGAA TTTGCGT-3', 18S rRNA forward 5'-TAACC CGTTGAACCCCATT-3' and reverse 5'-CCATCCAATCG GTAGTAGCG-3', and mature miR-27b forward 5'GAGCTTAGCTGATTGGTG-3' and universal reverse primer. 
Table I Clinical Data of Patients

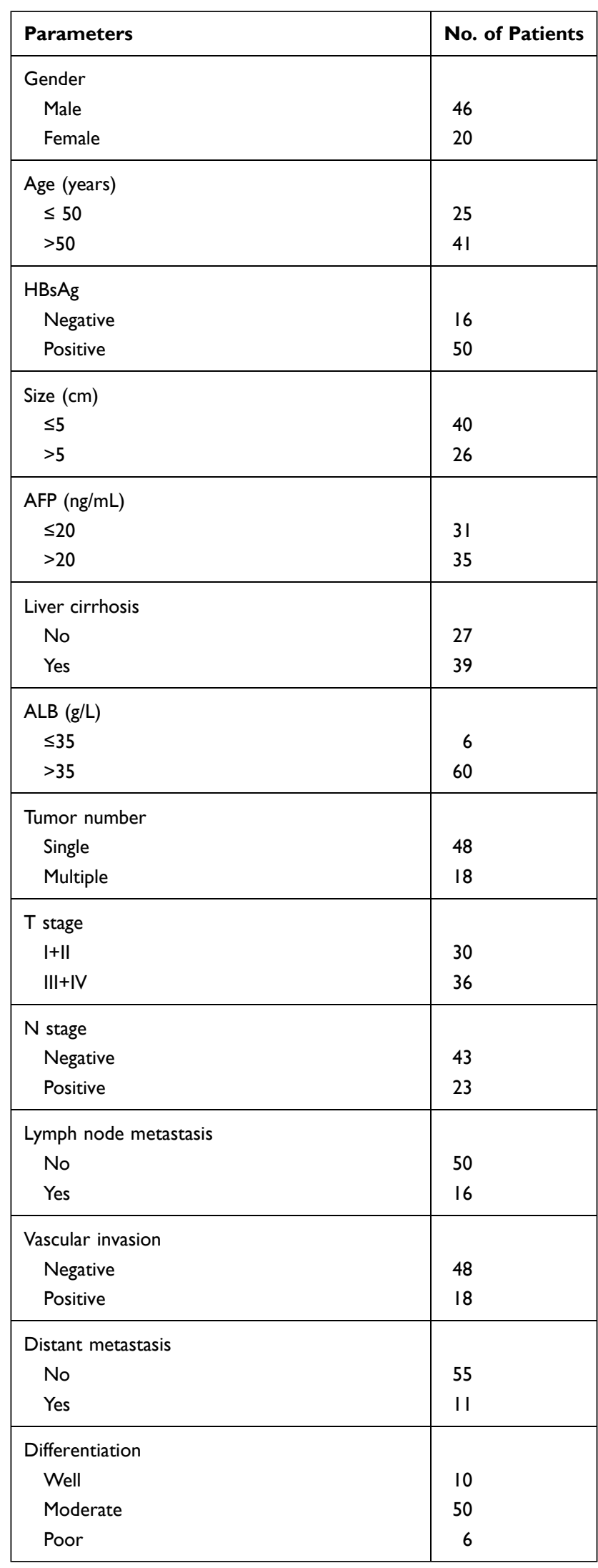

\section{RNA Pull-Down Assay}

Both DNAJC3-AS1 and negative control (NC) RNA in vitro transcripts were prepared using T7 RNA polymerase (NEB). DNAJC3-AS1 mutant, which was named DNAJC3-AS1 (mut), was prepared through site directed mutagenesis. Biotin-labeling was performed with Pierce ${ }^{\text {TM }}$ RNA 3' End Biotinylation Kit (Thermo Fisher Scientific), and the labeled RNAs were named Bio-DNAJC3-AS1 and Bio-NC, respectively. HepG2 and Huh7 cells were transfected with the two labeled RNAs, and cell lysis was performed 48h later on ice. Streptavidin Magnetic Beads (Thermo Scientific, USA) were used to pull down RNA complex. RNA isolation and RTqPCRs were performed following the aforementioned method to determine the expression of premature miR-27b.

\section{Subcellular Localization}

Both nucleus and cytoplasm samples of both HepG2 and Huh7 cells were prepared using Nuclear/Cytosol Fractionation Kit (Biovision). Cytoplasm sample was directly used for RNA isolation, while nucleus sample was further subjected to nucleus lysis, followed by RNA isolation. DNAJC3-AS1 expression in two samples were determined using RT-PCRs. PCR products were separated on $2 \%$ agarose gels, stained with ethidium bromide, and photographed and analyzed using MyECL imager.

\section{BrdU Assay}

Cell proliferation was evaluated by BrdU incorporation. In brief, cells were cultured in a 24 -well plate for $24 \mathrm{~h}$ after transfections, incubated with $10 \mu \mathrm{M}$ BrdU (SigmaAldrich) for another $2 \mathrm{~h}$, and fixed in $4 \%$ paraformaldehyde. Cells were then treated with $2 \mathrm{~N} \mathrm{HCl}$, washed with phosphate buffered saline (PBS), and incubation in turn with peroxidase-coupled anti-BrdU-antibody (SigmaAldrich) and peroxidase substrate. The OD values at 450 $\mathrm{nm}$ were determined using a plate reader.

\section{Statistical Analysis}

Paired $t$-test was used to compare paired tissue samples. Multiple ( $>2)$ independent groups and two independent groups were compared by ANOVA Tukey's test and unpaired $t$-test, respectively. The $66 \mathrm{HCC}$ patients were grouped into high and low DNAJC3-AS1 level groups ( $\mathrm{n}=33$, cutoff value = median level of DNAJC3-AS1 in HCC tissues). Follow-up data were used to plot survival curves, which were compared by log-rank $t$-test. $\mathrm{P}<0.05$ was statistical analysis. 
A

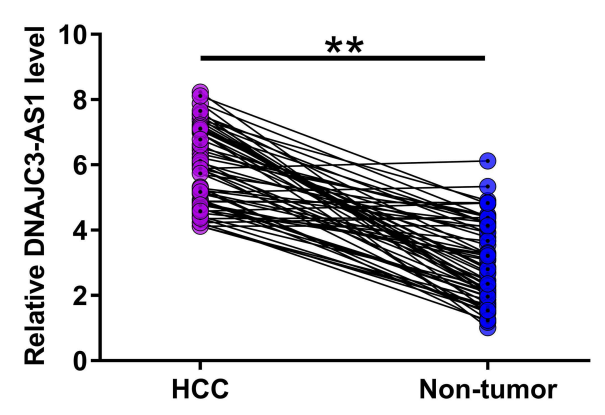

B

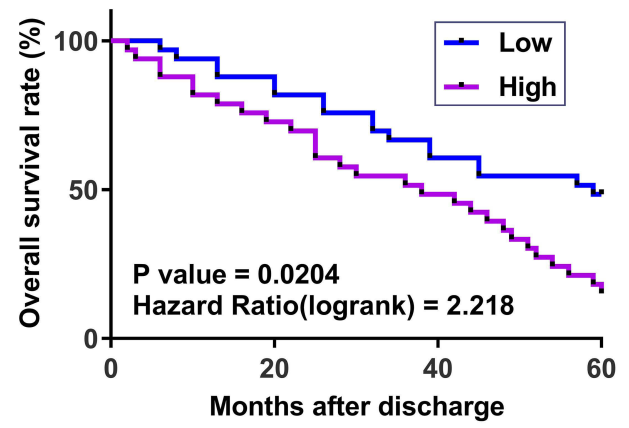

Figure I DNAJC3-ASI expression was upregulated in HCC and correlated with patients' prognosis. DNAJC3-ASI expression in 66 pairs of tissue samples donated by the 66 patients was analyzed with RT-qPCRs (A). Survival curve analysis was performed to analyze the prognostic value of DNAJC3-ASI for HCC (B). **P < 0.0I.

\section{Results}

\section{DNAJC3-ASI Expression Was} Upregulated in HCC and Correlated with Patients' Prognosis

DNAJC3-AS1 expression in 66 pairs of tissue samples donated by the 66 patients was analyzed with RT-qPCRs.

Our data analysis illustrated the increased expression of DNAJC3-AS1 in HCC tissues compared to non-tumor tissues, and the increase is about 1.82-fold (Figure 1A,

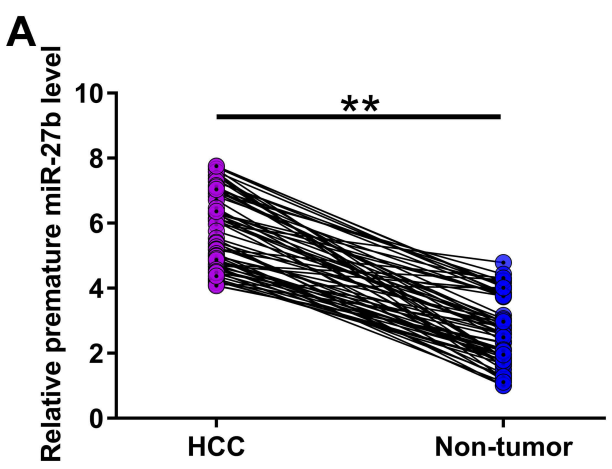

C

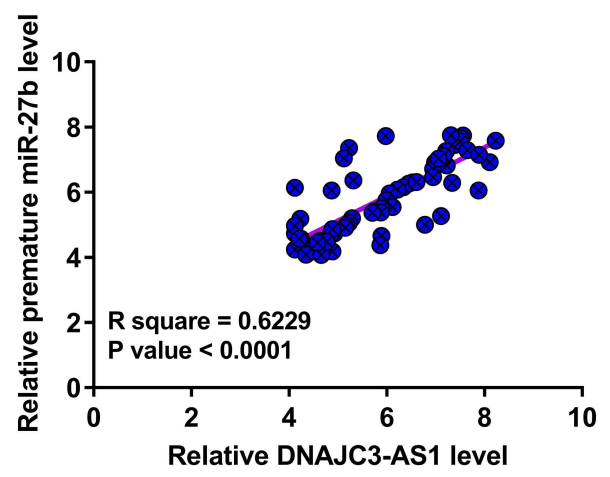

$\mathrm{p}<0.01)$. Survival curve analysis showed that high levels of DNAJC3-AS1 expression were closely correlated with the poor survival of HCC patients (Figure 1B). Therefore, DNAJC3-AS1 is likely a prognostic biomarker for HCC.

\section{Expression of Premature and Mature miR-27b Were Correlated with \\ DNAJC3-ASI in HCC}

MiR-27b expression at both premature and mature levels in 66 pairs of tissue samples donated by the 66 patients was

B

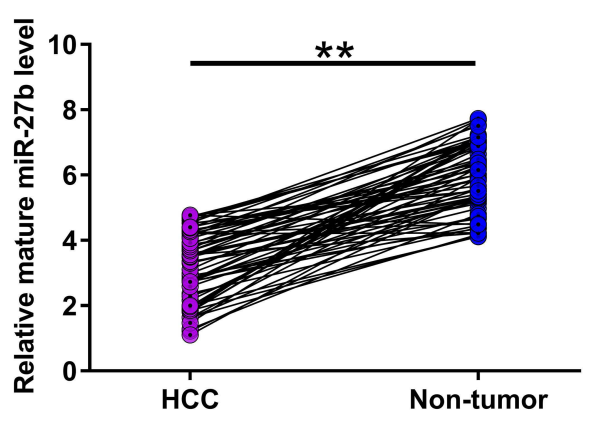

D

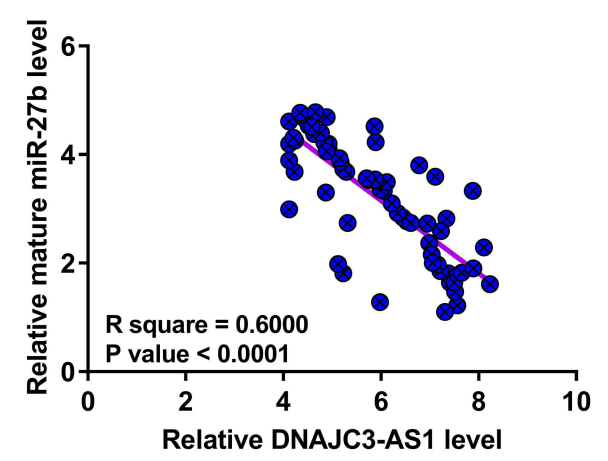

Figure 2 Expression of premature and mature miR-27b were correlated with DNAJC3-ASI in HCC. MiR-27b expression at both premature (A) and mature (B) levels in 66 pairs of tissue samples donated by the 66 patients was analyzed with RT-qPCRs. Correlations of premature (C) and mature miR-27b (D) with DNAJC3-ASI across HCC tissues were analyzed with Pearson's correlation coefficient. **p $<0.01$. 
analyzed with RT-qPCRs. Our data analysis showed that miR-27b was downregulated at mature miRNA level (1.99fold. Figure $2 \mathrm{~A}, \mathrm{p}<0.01$ ), but upregulated at premature level (1.72-fold, Figure 2B, $\mathrm{p}<0.01)$. Correlations of premature and mature miR-27b with DNAJC3-AS1 across HCC tissues were analyzed with Pearson's correlation coefficient, which showed that miR-27 was positively correlated with DNAJC3-AS1 at premature level (Figure 2C), but inversely correlated with DNAJC3-AS1 at mature level (Figure 2D).

\section{DNAJC3-ASI Was Associated Positively with Premature miR-27b in HCC Cells}

HepG2 and Huh7 cells were used to analyze the subcellular location of DNAJC3-AS1 through subcellular fractionation assay, which showed that DNAJC3-AS1 could be detected in both nucleus and cytoplasm samples (Figure 3A). The uncropped images are shown in Supplemental Figure 1. Analysis using IntaRNA 2.0 showed that DNAJC3-AS1 could form base pairs with premature miR-27b (Figure 3B). A DNAJC3-AS1 (mut), which was predicted unable to form base pairs with premature miR-27b, was prepared (indicated by the black letters in Figure 3A). RNA pull-down analysis showed that, compared to Bio-NC group, Bio-DNAJC3-AS1 group showed significantly higher level of premature miR$27 \mathrm{~b}$, suggesting a direct interaction between them (Figure $3 \mathrm{C}, \mathrm{p}<0.01$ ). In contrast, no significant difference in premature miR-27b level was observed between BioDNAJC3-AS1 (mut) and Bio-NC groups (Figure 3D).

\section{DNAJC3-ASI Regulated miR-27b Maturation}

HepG2 and Huh7 cells were transfected with DNAJC3-AS1 or miR-27b, and their expression was confirmed every $24 \mathrm{~h}$ until $72 \mathrm{~h}$. The results showed that their expression were increased more than 4-fold at $72 \mathrm{~h}$ (Figure 4A, $\mathrm{p}<0.05$; Figure $4 \mathrm{~B}, \mathrm{p}<0.05)$. In both cell lines, DNAJC3-AS1 overexpression increased premature miR-27b expression (Figure 4C, $\mathrm{p}<0.05$ ) and decreased mature miR-27b expression (Figure 4D, p < 0.05), while DNAJC3-AS1 knockdown by transfecting cells with siDNAJC3-AS1 (Figure 4E, $\mathrm{p}<0.05)$ downregulated premature miR-27b expression (Figure 4F, p $<0.05$ ) and upregulated mature miR-27b
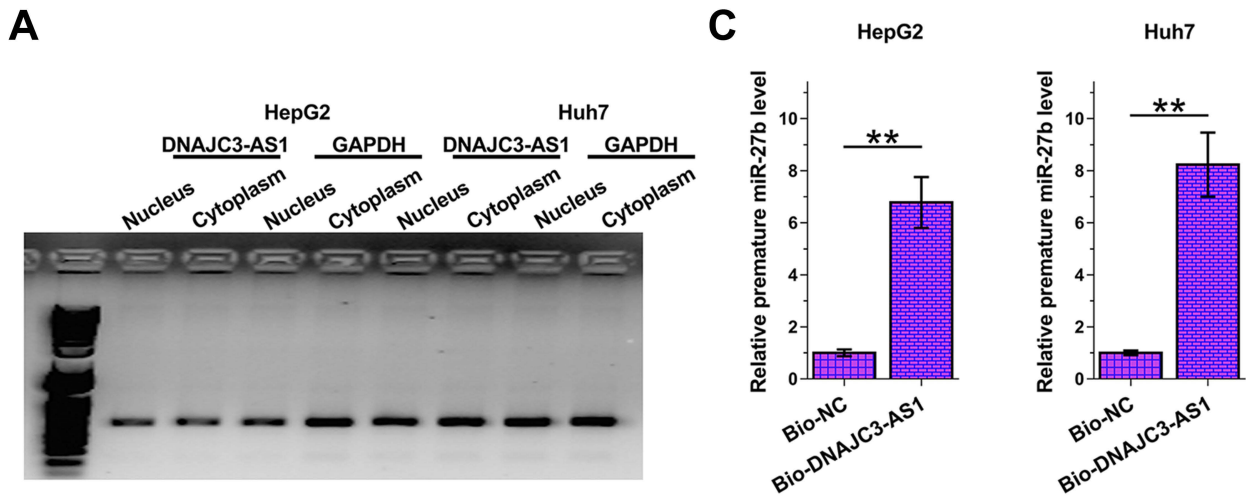

B

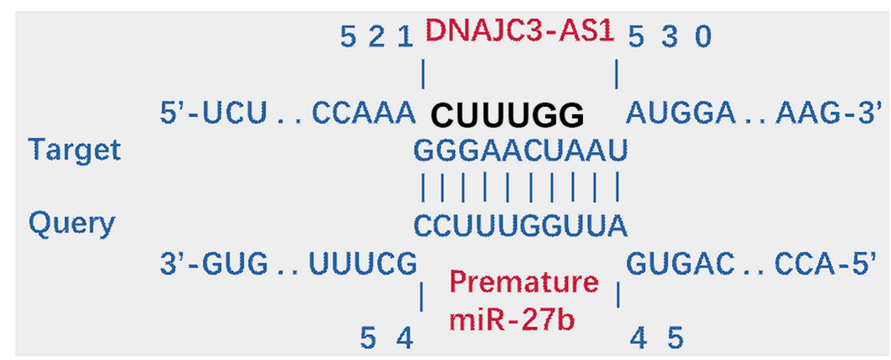

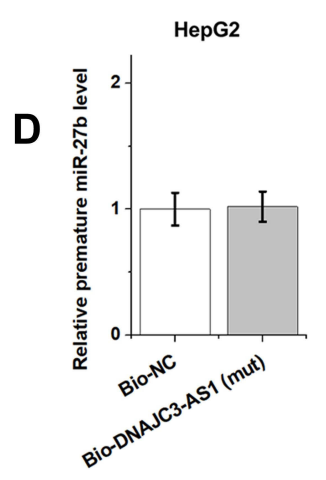

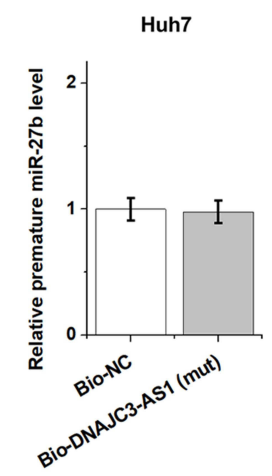

Figure 3 DNAJC3-ASI was associated positively with premature miR-27b in HCC cells. HepG2 and Huh7 cells were used to analyze the subcellular location of DNAJC3ASI through subcellular fractionation assay (A). IntaRNA 2.0 was used to predict the direct interaction between DNAJC3-ASI and premature miR-27b (B), which was confirmed by RNA pull-down assay (C). The interaction between premature miR-27b and Bio-DNAJC3-ASI (mut) was also analyzed by performing RNA-RNA pull-down assay (D). **p $<0.01$. 

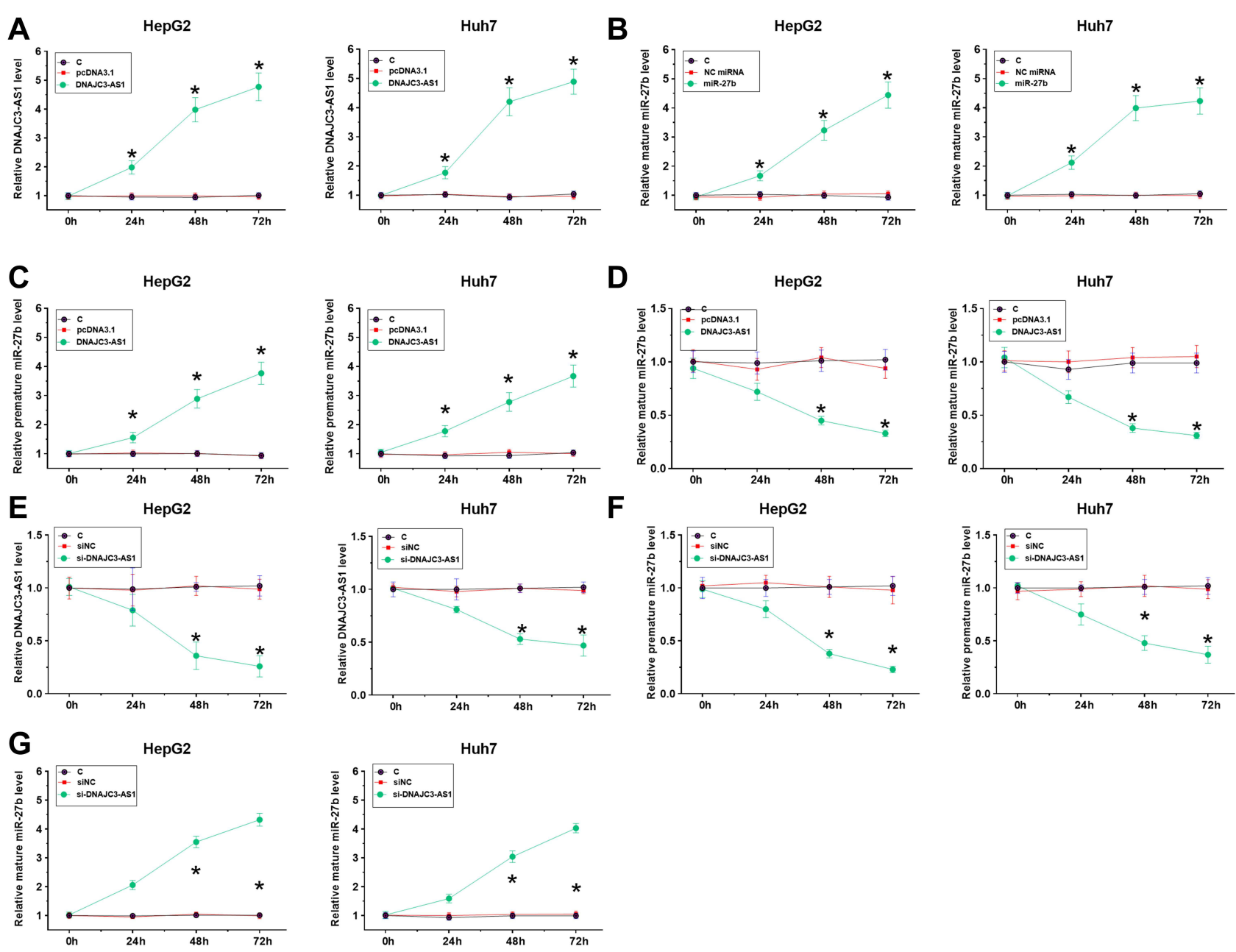

Figure 4 DNAJC3-ASI regulated miR-27b maturation. HepG2 and Huh7 cells were overexpressed with DNAJC3-ASI (A) or miR-27b (B). Their overexpression were confirmed every $24 \mathrm{~h}$ until $72 \mathrm{~h}$. The effect of DNAJC3-ASI overexpression on premature miR-27b (C) and mature miR-27b expression (D) and the effect of DNAJC3-ASI downregulation $(\mathbf{E})$ on premature miR-27b $(\mathbf{F})$ and mature miR-27b expression $(\mathbf{G})$ were detected by RT-qPCRs. ${ }^{*} \mathrm{p}<0.05$.

expression (Figure 4G, $\mathrm{p}<0.05$ ). Therefore, DNAJC3-AS inhibited miR-27b maturation.

\section{DNAJC3-ASI/miR-27b Axis Affected Cell Proliferation}

BrdU assay results revealed that DNAJC3-AS1 increased cell proliferation, miR-27b decreased cell proliferation, and DNAJC3-AS1 suppressed the inhibitory effect of miR-27b on cell proliferation (Figure 5A, $\mathrm{p}<0.05$ ). The role of DNAJC3-AS1(mut) in cell proliferation was also analyzed with BrdU assay. It was observed that DNAJC3-AS1(mut) failed to affect cell proliferation and the role of $\mathrm{miR}-27 \mathrm{~b}$ in cell proliferation (Figure 5B). Therefore, the role of DNAJC3-AS1 in cell proliferation is likely mediated by miR-27b.

\section{Discussion}

The present study explored the interaction between DNAJC3AS1 and miR-27b in HCC. We observed the altered DNAJC3AS1 expression and miR-27b maturation in HCC. Moreover, our data suggested the involvement of DNAJC3-AS1 in miR$27 \mathrm{~b}$ maturation, and the regulation of miR-27b maturation by DNAJC3-AS1 participates in HCC cell proliferation.

DNAJC3-AS1 is an oncogenic lncRNA characterized in several types of cancers. ${ }^{15-17}$ In colorectal cancer, its expression is increased. DNAJC3-AS1 overexpression interacts with epidermal growth factor receptor (EGFR) pathway to regulate fatty acid synthase, thereby promoting cancer progression. ${ }^{15}$ DNAJC3-AS1 expression is also increased in renal cell carcinoma, and it sponges miR-27a-3p to upregulate PRDM14, thereby accelerating cancer development. ${ }^{16}$ DNAJC3-AS1 interacts with its sense-cognate gene 
A

\section{HepG2}

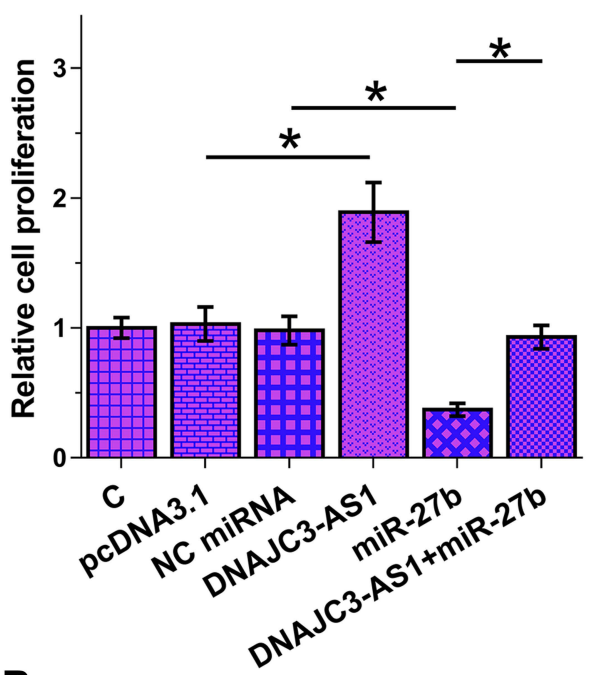

B

\section{HepG2}

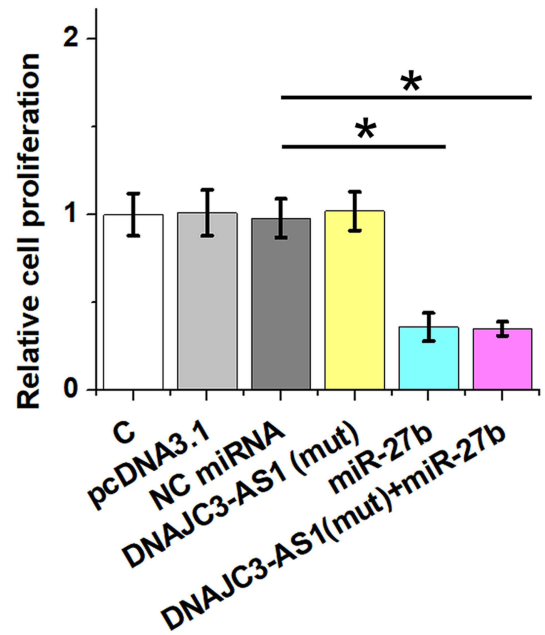

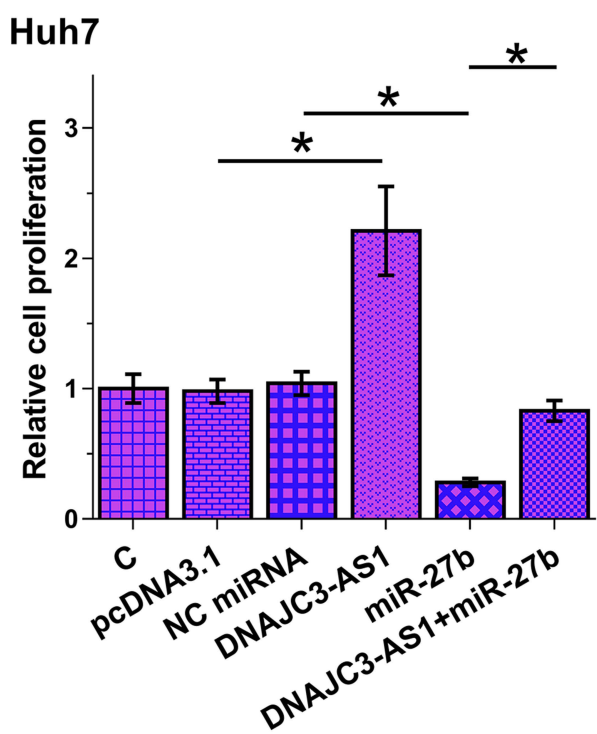

Huh7

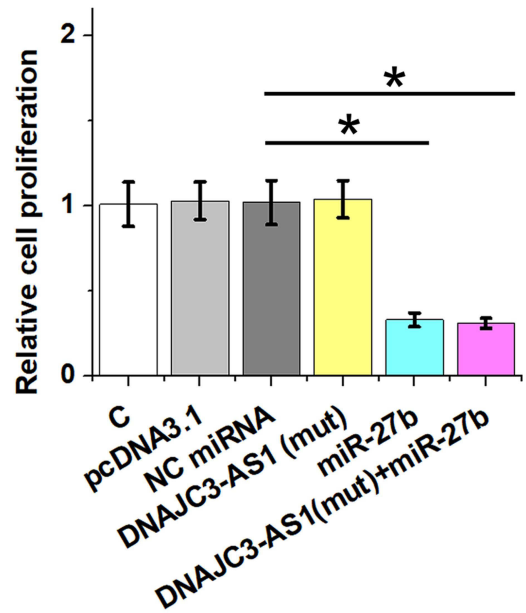

Figure 5 DNAJC3-ASI/miR-27b axis affected HCC cell proliferation. HepG2 and Huh7 cell proliferation after DNAJC3-ASI and/or miR-27b expression was analyzed with BrdU assays (A). The role of DNAJC3-ASI (mut) in cell proliferation was also analyzed with BrdU assay (B). *p $<0.05$.

DNAJC3 to participate in osteosarcoma. ${ }^{17}$ In the present study, we observed the upregulation of DNAJC3-AS1 in $\mathrm{HCC}$ and its role in enhancing cell proliferation. Therefore, DNAJC3-AS1 is likely an oncogenic lncRNA in HCC. Moreover, the present study also showed that high levels of DNAJC3-AS1 expression were closely correlated with the poor survival of HCC patients. Therefore, DNAJC3-AS1 upregulation may serve as a prognostic marker for HCC.

MiR-27b plays different roles in different types of cancers. ${ }^{18,19}$ For instance, miR-27b is lowly expressed in lung cancer and its overexpression targets snail 1 to inhibit lung cancer epithelial-mesenchymal transition, ${ }^{18}$ indicating its tumor suppressive roles. In contrast, a CRISPR/Cas9 deletion study revealed the oncogenic roles of miR-27b in cancer biology. ${ }^{19}$ However, the role of miR-27b in HCC is unclear. The present study revealed the downregulation of miR-27b in $\mathrm{HCC}$ and its inhibitory effect on HCC cell proliferation. Therefore, miR-27b is a tumor suppressor in HCC.

The key finding of the present study is that premature miR-27b in the nuclei of HCC cells could be sponged by DNAJC3-AS1, which were detected in both nuclear and cytoplasm fractions. In addition, DNAJC3-AS1 upregulated premature miR-27b and downregulated mature miR-27b. 
Therefore, DNAJC3-AS1 may sponge miR-27b in the nucleus to suppress its maturation, which requires the transportation of premature miR-27b from the nucleus to cytoplasm, thereby playing an oncogenic role in cell proliferation. This conclusion was supported by the experiments performed with mutated ASDNAJC3-AS1. DNAJC3-AS1 (mut) failed to bind premature miR-27b and showed no effect on cell proliferation. DNAJC3-AS1 (mut) also failed to significantly affect the role of miR-27b in cell proliferation.

This study reported a novel interaction between lncRNAs and miRNAs. DNAJC3-AS1 could sponge premature miR-27b to reduce the production of mature miR-27b. Although many approaches have been developed to treat $\mathrm{HCC},{ }^{20-26}$ the prognosis of HCC patients is still poor. The novel interaction between these two types of non-coding RNAs may provide novel insights to the studies of related human diseases. However, this study is limited by the small sample size. Conclusions from this study should be further verified. The interaction between DNAJC3-AS1 and premature miR-27b should also be verified by in vivo animal model experiments.

In conclusion, DNAJC3-AS1 is overexpressed in HCC and suppresses miR-27b maturation to promote $\mathrm{HCC}$ cell proliferation.

\section{Abbreviations}

lncRNA, Long non-coding RNA; HCC, hepatocellular carcinoma; miRNA, MicroRNA; FBS, foetal bovine serum; BrdU, 5-bromo-2'-deoxyuridine; EB, Ethidium bromide; PFA, Paraformaldehyde; OD, Optical density; RT-qPCR, Real Time Quantitative PCR; EGFR, Epidermal growth factor receptor.

\section{Data Sharing Statement}

The datasets generated during and/or analyzed during the current study are not publicly available, but are available from the corresponding author on reasonable request.

\section{Ethics Approval and Consent to Participate}

All patients signed informed consent. This study was approved by the Ethics Committee of the Edong Healthcare City Hospital of Traditional Chinese Medicine. All experiments were performed in accordance with the 1964 Declaration of Helsinki and its later amendments.

\section{Author Contributions}

All authors made a significant contribution to the work reported, whether that is in the conception, study design, execution, acquisition of data, analysis and interpretation, or in all these areas; took part in drafting, revising or critically reviewing the article; gave final approval of the version to be published; have agreed on the journal to which the article has been submitted; and agree to be accountable for all aspects of the work.

\section{Funding}

There is no funding to report.

\section{Disclosure}

The authors declare that they have no competing interests.

\section{References}

1. Yang JD, Hainaut P, Gores GJ, Amadou A, Plymoth A, Roberts LR. A global view of hepatocellular carcinoma: trends, risk, prevention and management. Nat Rev Gastroenterol Hepatol. 2019;16 (10):589-604. doi:10.1038/s41575-019-0186-y

2. McGlynn KA, Petrick JL, El-Serag HB. Epidemiology of hepatocellular carcinoma. Hepatology. 2021;73(Suppl 1):4-13.

3. Liu P, Xie SH, Hu S, et al. Age-specific sex difference in the incidence of hepatocellular carcinoma in the United States. Oncotarget. 2017;8(40):68131-68137. doi:10.18632/ oncotarget.19245

4. Bertuccio P, Turati F, Carioli G, et al. Global trends and predictions in hepatocellular carcinoma mortality. J Hepatol. 2017;67(2):302-309. doi:10.1016/j.jhep.2017.03.011

5. Beal EW, Tumin D, Kabir A, et al. Trends in the mortality of hepatocellular carcinoma in the United States. $J$ Gastrointest Surg. 2017;21(12):2033-2038. doi:10.1007/s11605-017-3526-7

6. Ganne-Carrié N, Chaffaut C, Bourcier V, et al. Estimate of hepatocellular carcinoma incidence in patients with alcoholic cirrhosis. J Hepatol. 2018;69(6):1274-1283. doi:10.1016/j.jhep.2018.07.022

7. Singh AK, Kumar R, Pandey AK. Hepatocellular carcinoma: causes, mechanism of progression and biomarkers. Curr Chem Genom Transl Med. 2018;12(1):9-26. doi:10.2174/2213988501812010009

8. Heimbach JK, Kulik LM, Finn RS, et al. AASLD guidelines for the treatment of hepatocellular carcinoma. Hepatology. 2018;67 (1):358-380. doi:10.1002/hep.29086

9. Daher S, Massarwa M, Benson AA, Khoury T. Current and future treatment of hepatocellular carcinoma: an updated comprehensive review. J Clin Transl Hepatol. 2018;6(1):69-78. doi:10.14218/ JCTH.2017.00031

10. Jindal A, Thadi A, Shailubhai K. Hepatocellular carcinoma: etiology and current and future drugs. J Clin Exp Hepatol. 2019;9(2):221-232. doi:10.1016/j.jceh.2019.01.004

11. Eso Y, Marusawa H. Novel approaches for molecular targeted therapy against hepatocellular carcinoma. Hepatol Res. 2018;48 (8):597-607. doi:10.1111/hepr.13181

12. Galun D, Srdic-Rajic T, Bogdanovic A, Loncar Z, Zuvela M. Targeted therapy and personalized medicine in hepatocellular carcinoma: drug resistance, mechanisms, and treatment strategies. J Hepatocell Carcinoma. 2017;4:93-103. doi:10.2147/JHC.S106529

13. Dai M, Chen S, Wei X, et al. Diagnosis, prognosis and bioinformatics analysis of lncRNAs in hepatocellular carcinoma. Oncotarget. 2017;8 (56):95799-95809. doi:10.18632/oncotarget.21329 
14. Xue C, Zhao Y, Jiang J, Li L. Expression levels of lncRNAs are prognostic for hepatocellular carcinoma overall survival. Am J Transl Res. 2020;12(5):1873-1883.

15. Tang Y, Tang R, Tang M, et al. LncRNA DNAJC3-AS1 regulates fatty acid synthase via the EGFR pathway to promote the progression of colorectal cancer. Front Oncol. 2020;10:604534. doi:10.3389/ fonc. 2020.604534

16. Na XY, Hu XQ, Zhao Y, Hu CH, Shang XS. LncRNA DNAJC3-AS1 functions as oncogene in renal cell carcinoma via regulation of the miR-27a-3p/PRDM14 axis. Eur Rev Med Pharmacol Sci. 2021;25 (3):1291-1301.

17. Liang R, Liu Z, Chen Z, et al. Long noncoding RNA DNAJC3-AS1 promotes osteosarcoma progression via its sense-cognate gene DNAJC3. Cancer Med. 2019;8(2):761-772. doi:10.1002/cam4.1955

18. Zhang J, Hua X, Qi $N$, et al. MiR-27b suppresses epithelial-mesenchymal transition and chemoresistance in lung cancer by targeting snail1. Life Sci. 2020;254:117238. doi:10.1016/j. lfs. 2019.117238

19. Hannafon BN, Cai A, Calloway CL, et al. miR-23b and miR-27b are oncogenic microRNAs in breast cancer: evidence from a CRISPR/ Cas9 deletion study. BMC Cancer. 2019;19(1):642. doi:10.1186/ s12885-019-5839-2

20. Chaiboonchoe A, Khraiwesh B, Murali C, et al. Safranal induces DNA double-strand breakage and ER-stress-mediated cell death in hepatocellular carcinoma cells. Sci Rep. 2018;8(1):16951. doi:10.1038/s41598-018-34855-0
21. Ashktorab H, Soleimani A, Singh G, et al. Saffron: the golden spice with therapeutic properties on digestive diseases. Nutrients. 2019;11 (5):943. doi:10.3390/nu11050943

22. Amin A, Mahmoud-Ghoneim D. Texture analysis of liver fibrosis microscopic images: a study on the effect of biomarkers. Acta Biochim Biophys Sin (Shanghai). 2011;43(3):193-203. doi:10.1093/ abbs/gmq129

23. Hamza AA, Heeba GH, Hamza S, et al. Standardized extract of ginger ameliorates liver cancer by reducing proliferation and inducing apoptosis through inhibition oxidative stress/inflammation pathway. Biomed Pharmacother. 2021;134:111102. doi:10.1016/j. biopha.2020.111102

24. Amin A, AlMansoori A, Baig B. Safranal-sorafenib combination therapy for liver cancer. U.S. Patent 10,568,873; 2020.

25. Amin A, Awad B. Crocin-sorafenib combination therapy for liver cancer. U.S. Patent 10,933,076; 2021.

26. Amin A. Prevention of liver cancer with safranal-based formulations. U.S. Patent 10,912,741; 2021

\section{Publish your work in this journal}

Cancer Management and Research is an international, peer-reviewed open access journal focusing on cancer research and the optimal use of preventative and integrated treatment interventions to achieve improved outcomes, enhanced survival and quality of life for the cancer patient.
The manuscript management system is completely online and includes a very quick and fair peer-review system, which is all easy to use. Visit http://www.dovepress.com/testimonials.php to read real quotes from published authors. 